\title{
Electromagnetic waves with negative phase velocity in Schwarzschild-de Sitter spacetime
}

\author{
Tom G. Mackay ${ }^{1}$ \\ School of Mathematics, University of Edinburgh, Edinburgh EH9 3JZ, UK \\ Akhlesh Lakhtakia ${ }^{2}$ \\ Department of Engineering Science and Mechanics \\ Pennsylvania State University, University Park, PA 16802-6812, USA \\ Sandi Setiawan ${ }^{3}$ \\ School of Mathematics, University of Edinburgh, Edinburgh EH9 3JZ, UK
}

\begin{abstract}
The propagation of electromagnetic plane waves with negative phase velocity (NPV) is considered in Schwarzschild-(anti-)de Sitter spacetime. It is demonstrated that NPV propagation occurs in Schwarzschild-de Sitter spacetime at lower values of the cosmological constant than is the case for de Sitter spacetime. Furthermore, we report that neither is NPV propagation observed in Schwarzschild-anti-de Sitter spacetime, nor is it possible outside the event horizon of a Schwarzschild blackhole.
\end{abstract}

\section{Introduction}

This communication concerns the propagation of electromagnetic planewaves with negative phase velocity (NPV) in curved spacetime. The phase velocity is called negative if the timeaveraged Poynting vector and the wavevector are oppositely aligned [1]. The phenomenon of negative refraction - which has lately generated considerable excitement in the electromagnetics and materials research communities [2] - follows as a direct consequence of NPV propagation. The prospect of technological applications, such as lenses with extremely low distortion, has prompted intense efforts by experimentalists and theoreticians directed towards the development of NPV-supporting artificial metamaterials [3].

The importance of NPV propagation in astrophysical scenarios has recently been emphasized. We have previously shown that vacuum, in association with certain spacetime metrics, can support NPV propagation [4, 5]. For example, the metric of de Sitter spacetime admits NPV propagation, whereas the anti-de Sitter metric does not [6]. Thus, an experimental means of distinguishing between anti-de Sitter and de Sitter spacetimes is offered

\footnotetext{
${ }^{1}$ Corresponding Author. Fax: + 44131650 6553; e-mail: T.Mackay@ed.ac.uk.

${ }^{2}$ Corresponding Author. Fax: +1 814863 4319; e-mail: akhlesh@psu.edu; also affiliated with Department of Physics, Imperial College, London SW7 2 BZ, UK

${ }^{3}$ Fax: + 44131650 6553; e-mail: S.Setiawan@ed.ac.uk.
} 
via NPV propagation. Furthermore, regions supporting NPV propagation are to be found within the ergosphere of a rotating black hole [7]. In this context, comparisons between NPV propagation and superradiant scattering are noteworthy. While both phenomenons involve negative energy densities, there are significant differences in terms of directional properties and frequency bounds [8].

In the present study we consider planewave propagation in Schwarzschild-(anti-)de Sitter spacetime. Our analysis is based on a formulation originally proposed by Tamm [9], wherein the formal analogy between electromagnetic propagation in gravitationally affected vacuum and propagation in a (fictitious) instantaneously responding medium is exploited [10] .

\section{Schwarzschild-(anti-)de Sitter spacetime}

The static Schwarzschild-(anti-)de Sitter metric, with the signature $(+,-,-,-)$, is conventionally expressed in spherical coordinates as $[11,12,13,14,15]$

$$
d s^{2}=(1-f) d t^{2}-\frac{1}{1-f} d r^{2}-r^{2}\left(d \theta^{2}+\sin ^{2} \theta d \phi^{2}\right)
$$

wherein $f=\left(2 G M r^{-1}+\Lambda r^{2} / 3\right) / c^{2}, M$ is the mass of the black hole, $c$ is the speed of light in vacuum in the absence of a gravitational field, $G$ is the gravitational constant, and $\Lambda$ is the cosmological constant. If the cosmological constant $\Lambda$ is positive, the spacetime is called Schwarzschild-de Sitter spacetime, whereas Schwarzschild-anti-de Sitter spacetime is characterized by $\Lambda<0$ [16]. Let us note that (1) reduces to

(a) the Schwarzschild metric when $\Lambda=0$, and

(b) the cosmological metric (de Sitter or anti-de Sitter, according to whether $\Lambda$ is positive or negative, respectively) when $M=0$.

In terms of Cartesian coordinates $x=r \sin \theta \cos \phi, y=r \sin \theta \sin \phi$, and $z=r \cos \theta$, the metric (1) is represented by $g_{\alpha \beta}$ as $^{4}$

$$
\left[g_{\alpha \beta}\right]=\left(\begin{array}{cccc}
1-f & 0 & 0 & 0 \\
0 & -1-\frac{f x^{2}}{r^{2}(1-f)} & -\frac{f x y}{r^{2}(1-f)} & -\frac{f x z}{r^{2}(1-f)} \\
0 & -\frac{f x y}{r^{2}(1-f)} & -1-\frac{f y^{2}}{r^{2}(1-f)} & -\frac{f y z}{r^{2}(1-f)} \\
0 & -\frac{f x z}{r^{2}(1-f)} & -\frac{f y z}{r^{2}(1-f)} & -1-\frac{f z^{2}}{r^{2}(1-f)}
\end{array}\right),
$$

\footnotetext{
${ }^{4}$ Roman indexes take the values 1, 2 and 3; while Greek indexes take the values 0, 1, 2, and 3 .
} 
and its inverse $g^{\alpha \beta}$ as

$$
\left[g^{\alpha \beta}\right]=\left(\begin{array}{cccc}
\frac{1}{1-f} & 0 & 0 & 0 \\
0 & -1+\frac{f x^{2}}{r^{2}} & \frac{f x y}{r^{2}} & \frac{f x z}{r^{2}} \\
0 & \frac{f x y}{r^{2}} & -1+\frac{f y^{2}}{r^{2}} & \frac{f y z}{r^{2}} \\
0 & \frac{f x z}{r^{2}} & \frac{f y z}{r^{2}} & -1+\frac{f z^{2}}{r^{2}}
\end{array}\right) .
$$

Following common practice $[10,17,18,19]$, the electromagnetic response of vacuum in curved spacetime may be described by the constitutive relations of an equivalent, instantaneously responding, medium as per

$$
\left.\begin{array}{l}
\underline{D}=\epsilon_{0} \underline{\underline{\gamma}} \cdot \underline{E} \\
\underline{B}=\mu_{0} \underline{\underline{\gamma}} \cdot \underline{H}
\end{array}\right\},
$$

wherein SI units are implemented. Here, $\epsilon_{0}=8.854 \times 10^{-12} \mathrm{~F} \mathrm{~m}^{-1}, \mu_{0}=4 \pi \times 10^{-12} \mathrm{H} \mathrm{m}^{-1}$, and $\underline{\underline{\gamma}}$ is the $3 \times 3$ dyadic equivalent of the metric $\left[\gamma_{a b}\right]$ with components

$$
\gamma_{a b}=-\frac{g^{a b}}{g_{00}} .
$$

\section{Piecewise uniform approximation}

A global description of Schwarzschild-(anti-)de Sitter spacetime is provided by the constitutive relations (4). Let us partition the global spacetime into adjoining neighbourhoods. At an arbitrary location $(\tilde{x}, \tilde{y}, \tilde{z})$, we consider the neighbourhood $\mathcal{R}$ which is taken to be sufficiently small that the nonuniform metric $\gamma_{a b}$ may be reasonably approximated by the uniform metric $\tilde{\gamma}_{a b}[7]$. By stitching together solutions from adjoining neighbourhoods, we formulate the global solution. This piecewise uniform approximation technique is commonly employed in solving differential equations with nonhomogeneous coefficients [20]. Thus, we have the uniform $3 \times 3$ dyadic representation

$$
\underline{\tilde{\gamma}} \equiv\left[\tilde{\gamma}_{a b}\right]=\frac{1}{1-\tilde{f}}\left(\begin{array}{ccc}
1-\frac{\tilde{f} \tilde{x}^{2}}{\tilde{r}^{2}} & -\frac{\tilde{f} \tilde{x} \tilde{y}}{\tilde{r}^{2}} & -\frac{\tilde{f} \tilde{x} \tilde{z}}{\tilde{r}^{2}} \\
-\frac{\tilde{f} \tilde{x} \tilde{y}}{\tilde{r}^{2}} & 1-\frac{\tilde{f} \tilde{y}^{2}}{\tilde{r}^{2}} & -\frac{\tilde{f} \tilde{y} \tilde{z}}{\tilde{r}^{2}} \\
-\frac{\tilde{f} \tilde{x} \tilde{z}}{\tilde{r}^{2}} & -\frac{\tilde{f} \tilde{y} \tilde{z}}{\tilde{r}^{2}} & 1-\frac{\tilde{f} \tilde{z}^{2}}{\tilde{r}^{2}}
\end{array}\right)
$$


at $\mathcal{R}$, with

$$
\tilde{f}=\frac{2 G M}{c^{2} \tilde{r}}+\frac{\Lambda \tilde{r}^{2}}{3 c^{2}}
$$

and $\tilde{r}^{2}=\tilde{x}^{2}+\tilde{y}^{2}+\tilde{z}^{2}$. We note that $\operatorname{det}[\underline{\tilde{\gamma}}]=(1-\tilde{f})^{-2}$.

\section{Plane waves in $\mathcal{R}$}

We seek planewave solutions

$$
\underline{E}=\operatorname{Re}\left\{\underline{E}_{0} \exp [i(\underline{k} \cdot \underline{r}-\omega t)]\right\}, \quad \underline{H}=\operatorname{Re}\left\{\underline{H}_{0} \exp [i(\underline{k} \cdot \underline{r}-\omega t)]\right\},
$$

to the source-free Maxwell curl postulates

$$
\nabla \times \underline{E}+\frac{\partial}{\partial t} \underline{B}=\underline{0}, \quad \nabla \times \underline{H}-\frac{\partial}{\partial t} \underline{D}=\underline{0}
$$

in $\mathcal{R}$. Here, the wavevector is denoted by $\underline{k}$ and the position vector within the neighbourhood containing $(\tilde{x}, \tilde{y}, \tilde{z})$ is represented by $\underline{r} ; \omega$ and $t$ are the angular frequency and time, respectively. Let us emphasize here that $\underline{r}$ and $t$ are independent of $\mathcal{R}$.

The amplitudes $\underline{E}_{0}$ and $\underline{H}_{0}$ are complex-valued with $i=\sqrt{-1}$. Upon combining (8) and (9), we find after some algebraic manipulation that

$$
\underline{\underline{W}} \cdot \underline{E}_{0}=\underline{0}
$$

where

$$
\underline{\underline{W}}=\left(k_{0}^{2} \operatorname{det}[\underline{\tilde{\gamma}}]-\underline{k} \cdot \underline{\tilde{\gamma}} \cdot \underline{k}\right) \underline{\underline{I}}+\underline{k} \underline{k} \cdot \underline{\tilde{\gamma}},
$$

and $k_{0}=\omega \sqrt{\epsilon_{0} \mu_{0}}$. Thus, the dispersion relation $\operatorname{det}[\underline{\underline{W}}]=0$ emerges, which can be recast as

$$
k_{0}^{2} \operatorname{det}[\underline{\tilde{\gamma}}]\left(k_{0}^{2} \operatorname{det}[\underline{\tilde{\gamma}}]-\underline{k} \cdot \underline{\tilde{\gamma}} \cdot \underline{k}\right)^{2}=0 .
$$

Clearly, the wavevectors must satisfy the condition

$$
\underline{k} \cdot \underline{\tilde{\gamma}} \cdot \underline{k}=k_{0}^{2} \operatorname{det}[\underline{\tilde{\gamma}}]
$$

if $\underline{\tilde{\gamma}}$ is nonsingular.

$=$ Let us consider now the eigensolutions of (10). In view of (13), we have

$$
\underline{k} \underline{k} \cdot \underline{\tilde{\gamma}} \cdot \underline{E}_{0}=\underline{0}
$$

hence, it follows that $\underline{E}_{0}$ is orthogonal to $\underline{k} \cdot \underline{\underline{\gamma}}$. The spacetime metric described by (1) is spherically symmetric. Therefore, there is no loss of generality in choosing the wavevector

$$
\underline{k}=k \underline{\hat{u}}_{z},
$$


with $\underline{\hat{u}}_{z}$ being the unit vector lying along the $z$ Cartesian axis. Thereby,

$$
\underline{k} \cdot \underline{\underline{\tilde{\gamma}}}=k\left(\tilde{\gamma}_{1} \underline{\hat{u}}_{x}+\tilde{\gamma}_{2} \underline{\hat{u}}_{y}+\tilde{\gamma}_{3} \underline{\hat{u}}_{z}\right)
$$

where

$$
\tilde{\gamma}_{1}=-\frac{\tilde{f} \tilde{x} \tilde{z}}{(1-\tilde{f}) \tilde{r}^{2}}, \quad \tilde{\gamma}_{2}=-\frac{\tilde{f} \tilde{y} \tilde{z}}{(1-\tilde{f}) \tilde{r}^{2}}, \quad \tilde{\gamma}_{3}=\frac{\tilde{f}_{z}}{(1-\tilde{f})}
$$

with

$$
\tilde{f}_{z}=1-\frac{\tilde{f} \tilde{z}^{2}}{\tilde{r}^{2}}
$$

and $\underline{\hat{u}}_{x}$ and $\underline{\hat{\hat{x}}}_{y}$ being unit vectors lying along the $x$ and $y$ Cartesian axes, respectively.

The two linearly independent eigenvectors

$$
\underline{e}_{1}=\tilde{\gamma}_{2} \underline{\hat{u}}_{x}-\tilde{\gamma}_{1} \underline{\hat{\hat{u}}}_{y}, \quad \underline{e}_{2}=\tilde{\gamma}_{1} \tilde{\tilde{\gamma}}_{3} \underline{\hat{\hat{u}}}_{x}+\tilde{\gamma}_{2} \tilde{\tilde{\gamma}}_{3} \underline{\hat{\underline{u}}}_{y}-\left(\tilde{\gamma}_{1}^{2}+\tilde{\gamma}_{2}^{2}\right) \underline{\hat{u}}_{z}
$$

satisfy (14); hence, we have the general solution

$$
\underline{E}_{0}=C_{1} \underline{e}_{1}+C_{2} \underline{e}_{2},
$$

with $C_{1}$ and $C_{2}$ being arbitrary complex-valued constants. The corresponding expression for $\underline{H}_{0}$ follows from the Maxwell postulates as

$$
\underline{H}_{0}=\frac{k}{\omega \mu_{0}}\left[C_{1}(1-\tilde{f}) \underline{e}_{2}-C_{2} \tilde{f}_{z} \underline{e}_{1}\right] .
$$

To calculate the wavenumbers we turn to the dispersion equation (13). For $\underline{k}$ aligned with $\underline{\hat{u}}_{z}$ we obtain the $k$-quadratic expression

$$
k^{2} \frac{\tilde{f}_{z}}{(1-\tilde{f})}-\frac{k_{0}^{2}}{(1-\tilde{f})^{2}}=0,
$$

from which the wavenumbers

$$
k= \pm k_{0}\left[(1-\tilde{f}) \tilde{f}_{z}\right]^{-1 / 2}
$$

straightforwardly emerge.

The requirement that $k \in \mathbb{R}$ imposes the condition

$$
(1-\tilde{f}) \tilde{f}_{z}>0
$$

in other words, both $(1-\tilde{f})$ and $\tilde{f}_{z}$ must have the same signs for propagating planewave solutions. 


\section{NPV Condition}

The propagation of planewaves with NPV is signalled by the inequality $[5,7]$

$$
\underline{k} \cdot\langle\underline{P}\rangle_{t}<0
$$

where $\langle\underline{P}\rangle_{t}=(1 / 2) \operatorname{Re}\left\{\underline{E}_{0} \times \underline{H}_{0}^{*}\right\}$ is the the time-averaged Poynting vector. The general solution (20) and (21) delivers

$$
\langle\underline{P}\rangle_{t}=\frac{k}{2 \omega \mu_{0}}\left[\left|C_{1}\right|^{2}(1-\tilde{f})+\left|C_{2}\right|^{2} \tilde{f}_{z}\right] \underline{e}_{1} \times \underline{e}_{2} .
$$

By virtue of (19), the orientation of $\langle\underline{P}\rangle_{t}$ is provided by the vector

$$
\underline{e}_{1} \times \underline{e}_{2}=\left(\tilde{\gamma}_{1}^{2}+\tilde{\gamma}_{2}^{2}\right)\left(\tilde{\gamma}_{1} \underline{\hat{u}}_{x}+\tilde{\gamma}_{2} \underline{\hat{u}}_{y}+\tilde{\gamma}_{3} \underline{\hat{u}}_{z}\right) \text {. }
$$

Thus,

$$
\underline{k} \cdot\left(\underline{e}_{1} \times \underline{e}_{2}\right)=k \frac{\tilde{f}}{1-\tilde{f}}\left[\frac{\tilde{f} \tilde{z}}{\tilde{r}^{2}(1-\tilde{f})}\right]^{2}\left(\tilde{x}^{2}+\tilde{y}^{2}\right)
$$

and it follows that

$$
\underline{k} \cdot\langle\underline{P}\rangle_{t}=\frac{1}{2 \omega \mu_{0}}\left[\frac{k \tilde{f} \tilde{z}}{\tilde{r}^{2}(1-\tilde{f})}\right]^{2}\left(\tilde{x}^{2}+\tilde{y}^{2}\right)\left(\left|C_{1}\right|^{2}+\left|C_{2}\right|^{2} \frac{\tilde{f}_{z}}{1-\tilde{f}}\right) \tilde{f}_{z} .
$$

Hence, by exploiting (24), we see that NPV arises as a consequence of $\tilde{f}_{z}<0$; i.e., NPV propagation occurs provided that

$$
\frac{6 G M}{\tilde{r}^{3}}+\Lambda>\frac{3 c^{2}}{\tilde{z}^{2}}
$$

Let us emphasize that while (30) has been derived for the neighbourhood $\mathcal{R}$, the location of $\mathcal{R}$ is arbitrary within Schwarzschild-(anti-)de Sitter spacetime. Therefore, the NPV inequality (30) applies generally.

In order to contextualize the NPV condition (30), it is helpful to introduce the event horizon for a Schwarzschild black hole which lies at $r=r_{\mathrm{Sch}}=2 G M / c^{2}$ and the event horizon for de Sitter spacetime which lies at $r=r_{\mathrm{deS}}=c \sqrt{3 / \Lambda}[21]$. Thereby, we see that (30) yields the following sufficient conditions for NPV propagation:

$$
\left.\begin{array}{ll}
\tilde{r}^{3}>r_{\mathrm{deS}}^{2}\left(\tilde{r}-r_{\mathrm{Sch}}\right) & \text { for } \quad \Lambda>0 \\
\tilde{r}^{3}<\frac{3 c^{2}}{\Lambda}\left(\tilde{r}-r_{\mathrm{Sch}}\right) & \text { for } \quad \Lambda<0
\end{array}\right\} .
$$




\section{Concluding remarks}

The general condition (30) has been derived for NPV propagation in Schwarzschild-(anti-)de Sitter spacetime. Analysis of this condition allows us to make the following conclusions:

(a) If $\Lambda=0$ (i.e., the spacetime is described by the Schwarzschild metric), then NPV propagation is indicated by the inequality

$$
\frac{r_{\mathrm{Sch}}}{\tilde{r}}>\frac{\tilde{r}^{2}}{\tilde{z}^{2}}
$$

Therefore, we deduce that NPV propagation is not possible outside the event horizon of a Schwarzschild black hole (i.e., for $\tilde{r}>r_{\mathrm{Sch}}$ ).

(b) If $M=0$ (i.e., the spacetime is (anti-)de Sitter), then NPV is signalled by the inequality

$$
\Lambda>\frac{3 c^{2}}{\tilde{z}^{2}}
$$

in agreement with earlier results [6]; i.e., anti-de Sitter spacetime does not support NPV propagation. Furthermore, we observe that it is the region of spacetime which lies outside the de Sitter event horizon which supports NPV propagation.

Let us analyze the NPV inequality (33) in view of the uniform approximation implemented in considering the neighbourhood $\mathcal{R}$. The linear dimensions $\delta$ of $\mathcal{R}$ are taken to be small relative to the global spacetime curvature and large compared to the electromagnetic wavelengths as given by $2 \pi / k$. Since the Ricci scalar $R$ - which provides a measure of the inverse radius of spacetime curvature squared - is given by $R=4 \Lambda / c^{2}$ for de Sitter spacetime [22], we have

$$
\frac{2 \pi}{|k|} \ll \delta \ll \frac{c}{2} \sqrt{\frac{\rho}{|\Lambda|}},
$$

with $\rho$ being a proportionality constant. Hence, the partition of global spacetime requires

$$
|\Lambda| \ll \frac{c^{2}|k|^{2} \rho}{16 \pi^{2}} .
$$

The inequalities (33) and (34) are mutually compatible as the linear dimensions $\delta$ of the neighbourhood $\mathcal{R}$ are chosen independently of the $\tilde{z}$ coordinate specifying the location of $\mathcal{R}$.

(c) By comparing (30) and (33), it is clear that in Schwarzschild--de Sitter spacetime NPV propagation occurs for smaller (positive) values of $\Lambda$ than is the case for de Sitter spacetime.

(d) Our numerical investigations have shown that the NPV inequality (30) is not satisfied for $\Lambda<0$ (i.e., Schwarzschild-anti-de Sitter spacetime) in physically probe-able regions of spacetime. 
Acknowledgments: SS acknowledges EPSRC for support under grant GR/S60631/01. As often, AL thanks the Mercedes Foundation for continuous support.

\section{References}

[1] LAKHTAKIA A., MCCALL M.W. and WEIGLHOFER W.S., Introduction to Complex Mediums for Optics and Electromagnetics, edited by W.S. WEIGLHOFER and A. LAKHTAKIA (SPIE Press, Bellingham, WA, USA) 2003, p.347.

[2] SHELBY R.A., SMITH D.R. and SCHULTZ S., Science, 292 (2001) 77.

[3] PENDRY J.B., Contemporary Phys., 45 (2004) 191.

[4] LAKHTAKIA A. and MACKAY T.G., J. Phys. A:Math. Gen., 37 (2004) L505; corrigendum 37 (2004) 12093.

[5] MACKAY T.G., LAKHTAKIA A. and SETIAWAN S., New J. Phys., 7 (2005) 75.

[6] MACKAY T.G., SETIAWAN S. and LAKHTAKIA A., Eur. Phys. J. C, (2005) DOI 10.1140/epjcd/s2005-01-001-9.

[7] LAKHTAKIA A., MACKAY T.G. and SETIAWAN S., Phys. Lett. A, 336 (2005) 89.

[8] SETIAWAN S., MACKAY T.G. and LAKHTAKIA A., Phys. Lett. A, 341 (2005) 15.

[9] TAMM I.E., Zhurnal Russkogo Fiziko-Khimicheskogo Obshchestva, Otdel Fizicheskii (J. Russ. Phys.-Chem. Soc, Phys. Section), 56 (1924) 248.

[10] SCHLEICH W. and SCULLY M.O., New Trends in Atomic Physics, edited by G. GRYNBERG and R. STORA (Elsevier Science Publishers, Amsterdam, Holland) 1984, p.995.

[11] GIBBOnS G.W. and HAWKInG S.W., Phys. Rev. D, 15 (1977) 2738.

[12] BOUsso R. and HAWKInG S.W., Phys. Rev. D, 57 (1998) 2436.

[13] PODOLSKY J., Gen. Rel. Grav., 31 (1999) 1703.

[14] CARdoso V. and LEMOS J.P.S., Phys. Rev. D, 65 (2002) 104033.

[15] CARdoso V. and LEMOS J.P.S., Phys. Rev. D, 64 (2001) 084017.

[16] STEPHANI H., KRAMER D., MACCALLUM M., HOENSELAERS C. and HERLT E., Exact Solutions of Einstein's Field Equations, 2nd ed (Cambridge University Press, Cambridge, UK) 2003, chapt. 8.

[17] SKROTSKII G.V., Soviet Phys.-Dokl., 2 (1957) 226. 
[18] PLEBANSKI J., Phys. Rev., 118 (1960) 1396.

[19] BINI D., CHERUBINI C. and MASHHOON B., Class. Quantum Grav., 21 (2004) 3893.

[20] HOFFMAN J.D., Numerical Methods for Engineers and Scientists (McGraw-Hill, New York, USA) 1992.

[21] D'INVERNO R., Introducing Einstein's Relativity, (Clarendon Press, Oxford, UK) 1992.

[22] PeEBles P.J.E., Principles of Physical Cosmology, (Princeton University Press, Princeton, NJ, USA) 1993. 